\title{
Mandibular angle reduction combined with facelift via the premasseter space
}

\author{
Yoon Joo Lee ${ }^{1}$, Il Seok Lee ${ }^{2}$, \\ Ho Jik Yang ${ }^{2}$ \\ ${ }^{1}$ Doctorsmi Aesthetic Plastic Surgical \\ Clinic, Daejeon; ${ }^{2}$ Department of Plastic \\ and Reconstructive Surgery, Eulji \\ University Hospital, Daejeon, Korea
}

This article was presented as a free paper presentation at PRS Korea 2019 on November 8, 2019, in Seoul, Korea.

\begin{abstract}
Background In Asian women who undergo facelift procedures, satisfying results are achieved for facial rhytides, but sometimes there are postoperative complaints concerning the mandibular angle. Unlike Caucasians, Asians generally have a prominent mandibular angle. Accordingly, bone contouring surgery must be considered, since the bones of the face serve as the frame for facelift surgery. We investigated the effects of simultaneously performing mandibular angle reduction and facelift to achieve an oval facial contour and a youthful face.

Methods We evaluated 17 Asian women who simultaneously underwent mandibular angle reduction and facelift between April 2016 and May 2018. The clinical results were assessed based on preoperative and postoperative photographs and the Global Aesthetic Improvement Scale.

Results Surgery was successful in all cases. Postoperatively, improvements in facial rhytides and appropriate mandibular contours were achieved. All patients were satisfied with the outcomes. Some patients experienced short-term complications, such as hematoma and numbness of the skin above the incision line; however, these complications improved. Serious long-term complications were not noted.

Conclusions Highly satisfying outcomes can be achieved with combined mandibular angle reduction and facelift for Asian women with a wide and rectangular face.
\end{abstract}

Keywords Facelift / Mandible / Angle / Osteotomy

\section{INTRODUCTION}

The demand for facial rejuvenation surgery is increasing with the increase in the elderly population. Facelift surgery is widely used to lift the soft tissue, thereby weakening the nasolabial groove and providing volumetric fullness to the face [1]. However, owing to differences in anatomical structures between Asian and Caucasian faces, this method does not always result in satisfying outcomes for

Received: Jun 16, 2020 Revised: Jul 31, 2020 Accepted: Aug 11, 2020 Correspondence: Ho Jik Yang Department of Plastic and Reconstructive Surgery, Eulji University Hospital, 95 Dunsanseo-ro, Seo-gu, Daejeon 35233, Korea

Tel: +82-42-611-3029, Fax: +82-42-259-1111, E-mail: drhjyang@gmail.com

Copyright $\odot 2020$ The Korean Society for Aesthetic Plastic Surgery.

This is an Open Access article distributed under the terms of the Creative Commons Attribution Non-Commercial License (https://creativecommons.org/licenses/by-nc/4.0/) which permits unrestricted non-commercial use, distribution, and reproduction in any medium, provided the original work is properly cited. www.e-aaps.org all Asian women. Asian faces differ from Caucasian faces with respect to the skull structure and distribution of facial fat. Compared with Caucasians, Asians generally have wider and shorter faces. Facial fat descends with age, and Asian faces become more rectangular and boxy $[2,3]$. For the aforementioned reasons, some old Asian women have a prominent mandibular angle and a face with a rectangular appearance. In these patients, performing only a soft tissue facelift will cause the face to look more rectangular and the mandibular angle to appear more protruded.

In Asian cultures, an overly prominent mandible and rectangular face are undesirable because they suggest a harsh and masculine appearance. Instead, facial beauty is characterized by an oval appearance. Patients with a prominent mandibular angle often complain that their face looks more rectangular after surgery when only a facelift is performed without consideration of this cultural difference.

Therefore, in light of the cultural and anatomical differences between Asians and Caucasians, lifting soft tissue alone does not pro- 
vide satisfactory results for Asian women with a prominent mandibular angle.

To resolve this issue, we performed mandibular angle reduction during facelift procedures to achieve an oval face. We report this surgical technique and discuss its effects.

\section{METHODS}

This study included 17 Asian women who simultaneously underwent mandibular angle reduction and facelift between April 2016 and May 2018. The mean age of the patients was 54 years; smokers and patients with concomitant medical illnesses were excluded.

The study protocol was reviewed and approved by the institutional review board (approval No. EMC 2019-12-004-001). Informed consent was provided by all subjects before enrollment.

All surgical procedures were performed under general anesthesia through orotracheal intubation. To reduce intraoperative bleeding, surgery was performed approximately 15 minutes after administration of tumescent solution. After making an incision that followed the temporal hairline, tragal margin, and occipital hairline, the skin flap was elevated. To release the subcutaneous attachment of crow's feet and to achieve smooth redraping of the temporal skin, subcutaneous dissection was performed above the zygomatic arch of the lateral orbital area. A vertical incision was made on the superficial musculoaponeurotic system (SMAS) $1 \mathrm{~cm}$ anterior to the preauricular skin incision, followed by SMAS dissection. By following the dissection towards the anterior and inferior directions of the SMAS, the orbicularis oculi and platysma were connected and included in the SMAS flap. The main zygomatic and upper masseteric retaining ligaments were carefully released to avoid facial nerve injury.

After sufficient elevation of the SMAS flap, the premasseter space between the platysma and masseter fascia was approached. The lower half of the premasseter space is an avascular cleavage plane, which contains no vital structures. The mandible can be approached by dissection of the masseter fascia and muscle between the buccal branch and upper mandibular branch of facial nerve using this space, depending on the shape of the mandibular bone and amount of bone to be resected (Fig. 1).

A horizontal line of about $2 \mathrm{~cm}$ can be made on the masseter fascia between the buccal branch and the upper mandibular branch of the facial nerve. The line must be located at least $15 \mathrm{~mm}$ above the mandibular rim near the mandibular angle, comfortably superior to the mandibular branch of the facial nerve [4]. When the masseter fascia was exposed, the fascia was dissected using small Metzenbaum scissors at a distance from the facial nerve with the naked eye. The masseter muscle was then split and subperiosteal dissection of the mandible was performed. After sufficient subperiosteal dissection, mandibular angle cutting was done carefully to avoid soft tissue injury. It is important to ensure that the fa-

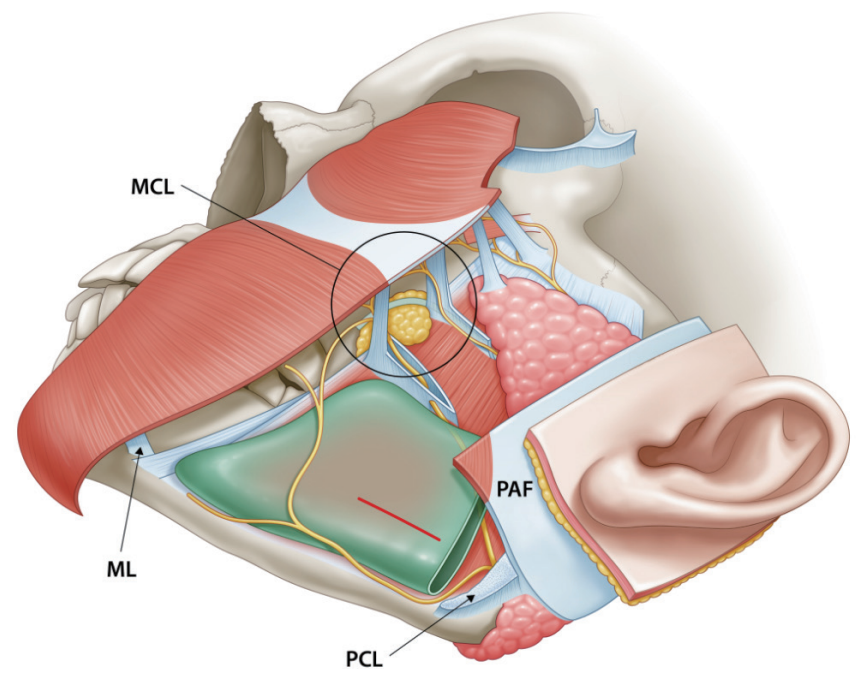

Fig. 1. Anatomy of the premasseter space. The premasseter space is located in the lateral lower third of the face between the platysma and the masseter fascia, and is made up by the masseteric cutaneous ligament (upper anterior), the mandibular ligament (lower anterior), the posterior edge of the platysma auricular fascia (posterior), and a mesenteric-like structure without any ligaments (inferior). The red horizontal line is the line for dissection on the masseter fascia for a subperiosteal flap. Using this line, the mandible can be approached by dissection on the masseter fascia and muscle between the buccal branch and the upper mandibular branch of the facial nerve. $M C L$, masseteric cutaneous ligament; ML, mandibular ligament; PAF, platysma auricular fascia; PCL, parotid cutaneous ligament.

cial nerve branches are not injured and to reduce soft tissue injury to prevent postoperative hematoma. Through this space, the mandibular angle was exposed and a reciprocating saw was used to perform mandibular angle reduction (Fig. 2).

After completing mandibular angle reduction, the SMAS flap was redraped on the cheek. The SMAS flap was pulled parallel to the long axis of the zygomaticus major and to the edge of the original SMAS incision. The redundant tissue was then removed. The preauricular SMAS flap was postauricularly transposed and fixed to the mastoid fascia. The cheek skin flap was horizontally redraped, and the redundant skin was removed. Skin closure was performed with minimal to no tension. A negative suction drainage catheter was used, and a gentle-compression dressing was applied.

The postoperative results were assessed using preoperative and postoperative photographs and Global Aesthetic Improvement Scale (GAIS) scores (Table 1).

\section{RESULTS}

Seventeen patients underwent mandibular angle reduction with facelift. The median operative time was 225 minutes (range, 200245 minutes). The patients were discharged at 1 day postoperative- 

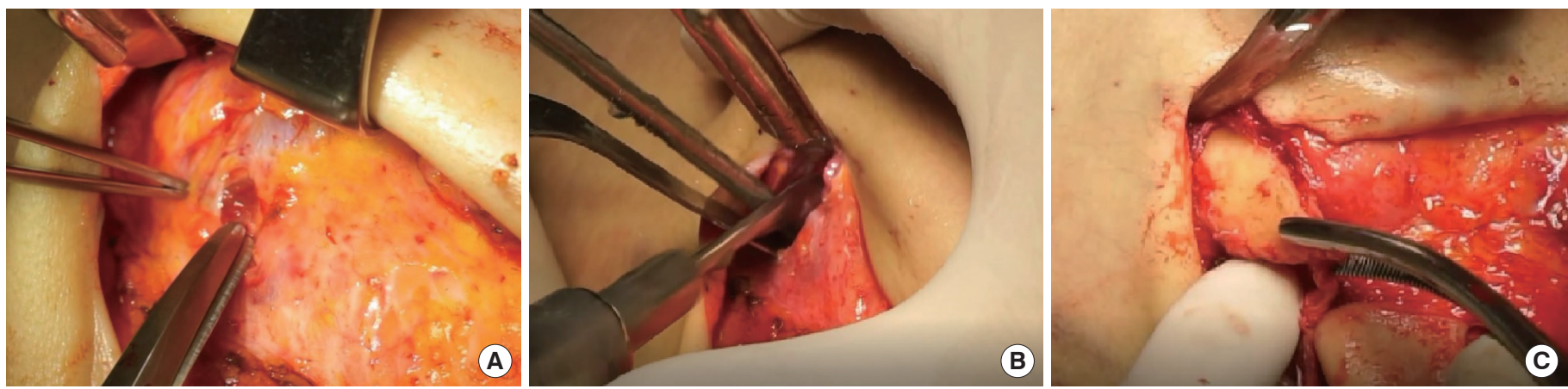

Fig. 2. Facial bone contouring. (A) The masseter fascia was dissected using small Metzenbaum scissors for subperiosteal dissection. (B) Ostectomy of the mandibular angle via the premasseter space after subperiosteal dissection of the masseter muscle. (C) As shown in the picture, the mandibular angle was easily visualized and removed through approximately 2-cm-long incision.

Table 1. Global Aesthetic Improvement Scale scores

\begin{tabular}{ll}
\hline Degree & \multicolumn{1}{c}{ Description } \\
\hline $\begin{array}{l}\text { 1 Exceptional improvement } \\
2 \text { Very improved patient }\end{array}$ & $\begin{array}{c}\text { Excellent corrective result } \\
\text { Marked improvement of the appearance, but } \\
\text { not complete } \\
\text { Improvement of the appearance, better com- } \\
\text { pared with the initial condition, but a touch- } \\
\text { up is advised }\end{array}$ \\
$\begin{array}{l}\text { The appearance substantially remains the } \\
\text { same compared with the original condition }\end{array}$ \\
$\begin{array}{l}\text { The appearance has worsened compared with } \\
\text { the original condition }\end{array}$
\end{tabular}

ly. They were examined at 3 days postoperatively. The sutures were removed on postoperative day 7 . Routine follow-up was performed at 3 and 6 months postoperatively.

All patients were satisfied with the results, and there were no serious complications. Side effects such as unexpected bone fracture or injury of the facial nerve were not noted. One patient experienced hematoma, and revision was performed for bleeding control. After a proper revision, satisfying results were obtained. Two patients experienced numbness of the skin above the incision line, but the symptoms had improved by 6 months postoperatively. All patients were preoperatively informed that they may experience numbness as a side effect. Surgical outcomes were assessed using the GAIS score. Four (23.5\%) patients had a GAIS score of 1, eight $(47 \%)$ had a score of 2 , and five $(29.5 \%)$ had a score of 3 (Table 2 , Figs. 3-5).

\section{DISCUSSION}

The aim of facelift procedures is not only to remove wrinkles via simple skin tightening, but also to achieve a three-dimensionally young-looking face. Accordingly, the sagging of the soft tissue as a consequence of aging and its association with the facial contour
Table 2. Assessments of surgical outcomes by Global Aesthetic Improvement Scale scores

\begin{tabular}{|c|c|c|}
\hline Degree & Description & $\begin{array}{c}\text { No. of } \\
\text { patients }(\%)\end{array}$ \\
\hline 1 Exceptional improvement & Excellent corrective result & 4 (23.5) \\
\hline 2 Very improved patient & $\begin{array}{l}\text { Marked improvement of the } \\
\text { appearance, but not complete }\end{array}$ & $8(47.0)$ \\
\hline 3 Improved patient & $\begin{array}{l}\text { Improvement of the appearance, } \\
\text { better compared with the initial } \\
\text { condition, but a touch-up is } \\
\text { advised }\end{array}$ & 5 (29.5) \\
\hline 4 Unaltered patient & $\begin{array}{l}\text { The appearance substantially } \\
\text { remains the same compared } \\
\text { with the original condition }\end{array}$ & 0 \\
\hline 5 Worsened patient & $\begin{array}{l}\text { The appearance has worsened } \\
\text { compared with the original } \\
\text { condition }\end{array}$ & 0 \\
\hline
\end{tabular}

should be considered. With aging, the face becomes wide due to facial bony changes; facial width increases and bony prominences become coarser $[5,6]$. Satisfactory results cannot be obtained if only a facelift is performed, without considering the facial bony changes that occur with aging.

Anatomically, compared with Caucasians, Asians have thicker skin and a wider and shorter facial geometry $[7,8]$. Asians have a distinct facial contour, involving a brachycephalic cranium with a prominent mandibular angle. These features results in a short, wide and rectangular facial contour, which is considered to be unaesthetic and masculine in Asian cultures, where facial beauty is characterized by an oval appearance. Patients with a prominent mandibular angle often complain that their face looks more rectangular after surgery when only a facelift is performed, without considering this cultural difference. It is difficult to obtain satisfying results through simple facial rejuvenation in patients with such features. A more prominent mandibular angle following rhytides improvement is a complaint after facelift in some Asian women 

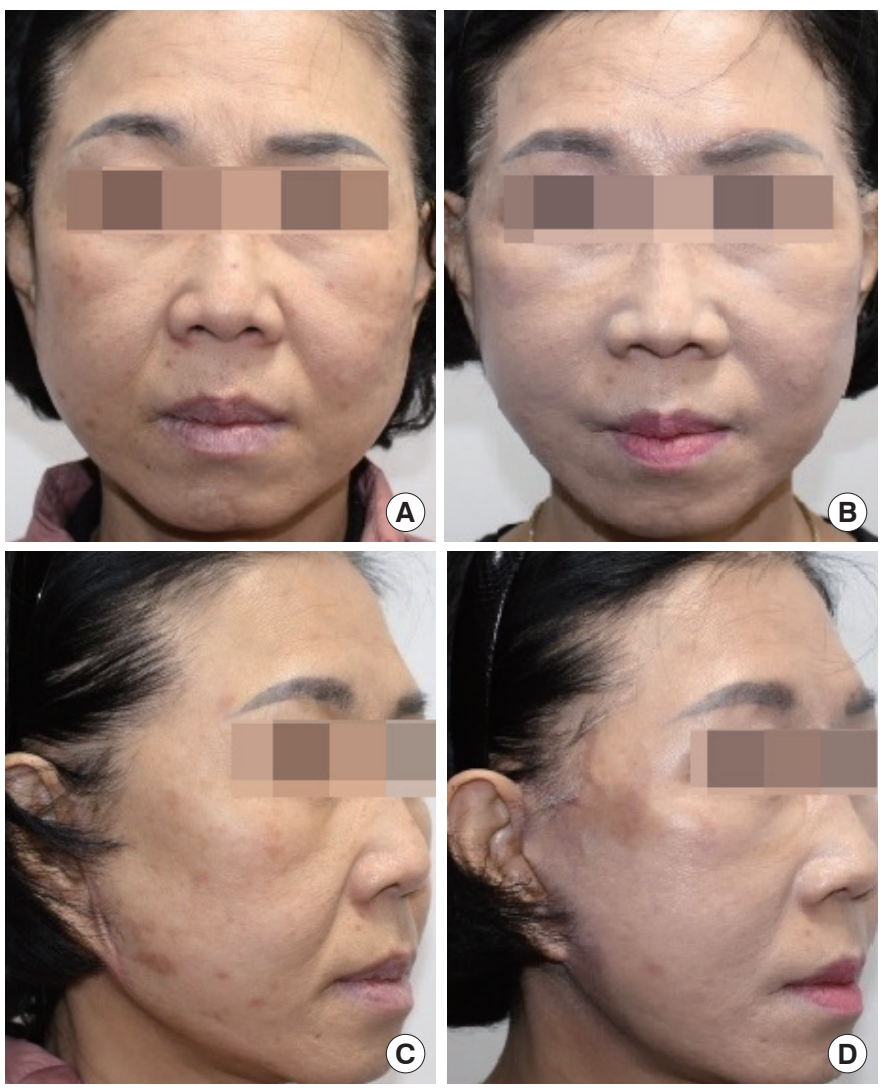

Fig. 3. A 58-year-old woman underwent mandibular angle reduction with facelift surgery. Preoperatively, she showed a pixie ear deformity and hypertrophic scar at the surgical site as complications from facelift surgery 8 years ago. $(A, C)$ Preoperative photograph. $(B, D)$ Postoperative photograph 6 months later, in which the facial rhytides and mandibular contour had significantly improved. The malar fat pad was lifted, and reduction of the width of the lower face can be observed. The patient was very satisfied with the results.

with a wide jaw. Therefore, without considering cultural and anatomical differences between Asians and Caucasians, lifting soft tissue alone does not provide satisfactory results.

Although methods for facial rejuvenation such as implant insertion and volumetric restoration using fat grafting are available $[9,10]$, these methods are associated with complications such as infection, implant displacement, bony absorption, and contour irregularity. The facelift technique suspends subcutaneous mandibular and malar fat masses in the cheek area; therefore, it can effectively improve facial rhytides and long-lasting results can be achieved [11]. It is clear that facelift can reduce the depth of the nasolabial fold and improve both the jowl and the jawline to achieve a more youthful appearance. In order to achieve more effective aesthetic results, it has recently been pointed out that skeletal support is important for soft tissue repositioning; therefore, the facial skeleton should be considered before soft-tissue repositioning [12]. It should be kept in mind that the primary goal of facelift procedures
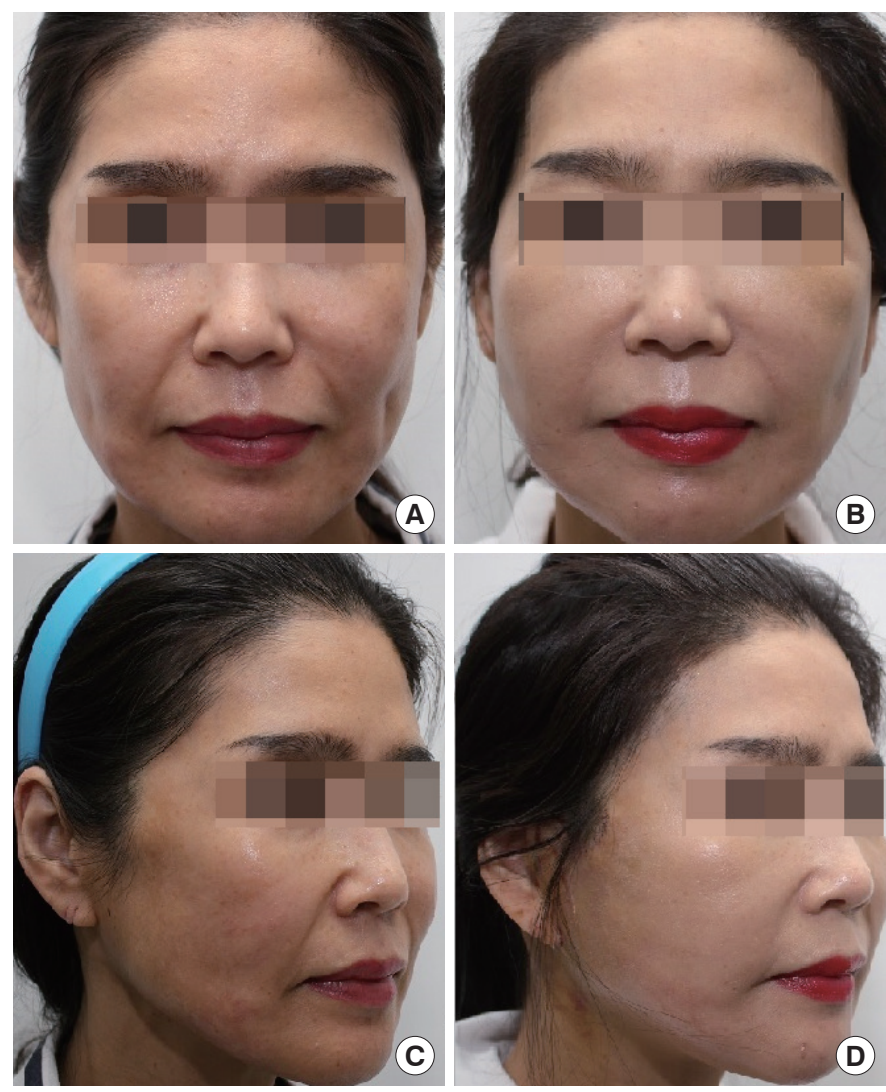

Fig. 4. A 46-year-old woman underwent mandibular angle reduction with facelift surgery. (A, C) Preoperative photograph. (B, D) Postoperative photograph 6 months later, in which the facial rhytides and mandibular contour had significantly improved. Weakening of the nasolabial fold and the change from a rectangular face to an oval face can be observed in the postoperative photograph.

is to improve the overall facial appearance, just not to improve rhytides. Especially in Asian women with a prominent mandibular angle, it is difficult to obtain an attractive facial contour without considering bony support. We attempted to reduce the mandibular angle in patients with a wide face to achieve a more attractive facial appearance.

The presence of a prominent mandibular angle in Asian women suggests an unaesthetic and masculine appearance. This led to the development of procedures to achieve an aesthetically pleasing mandibular contour. A rectangular face occurs in Asians not only due to hypertrophy of the masseter muscle, but also to the prominent mandibular angle. Patients with a hypertrophic masseter muscle can temporarily solve this problem through a Botox injection. However, many Asians with a prominent mandibular angle want to improve their rectangular face by reducing the size of their prominent mandibular angle. Therefore, mandibular angle reduction surgery is frequently performed in Asians to reduce the width of the lower face and to change a rectangular face to an oval face. Facelift alone cannot bring dramatic effects in the mandibular an- 


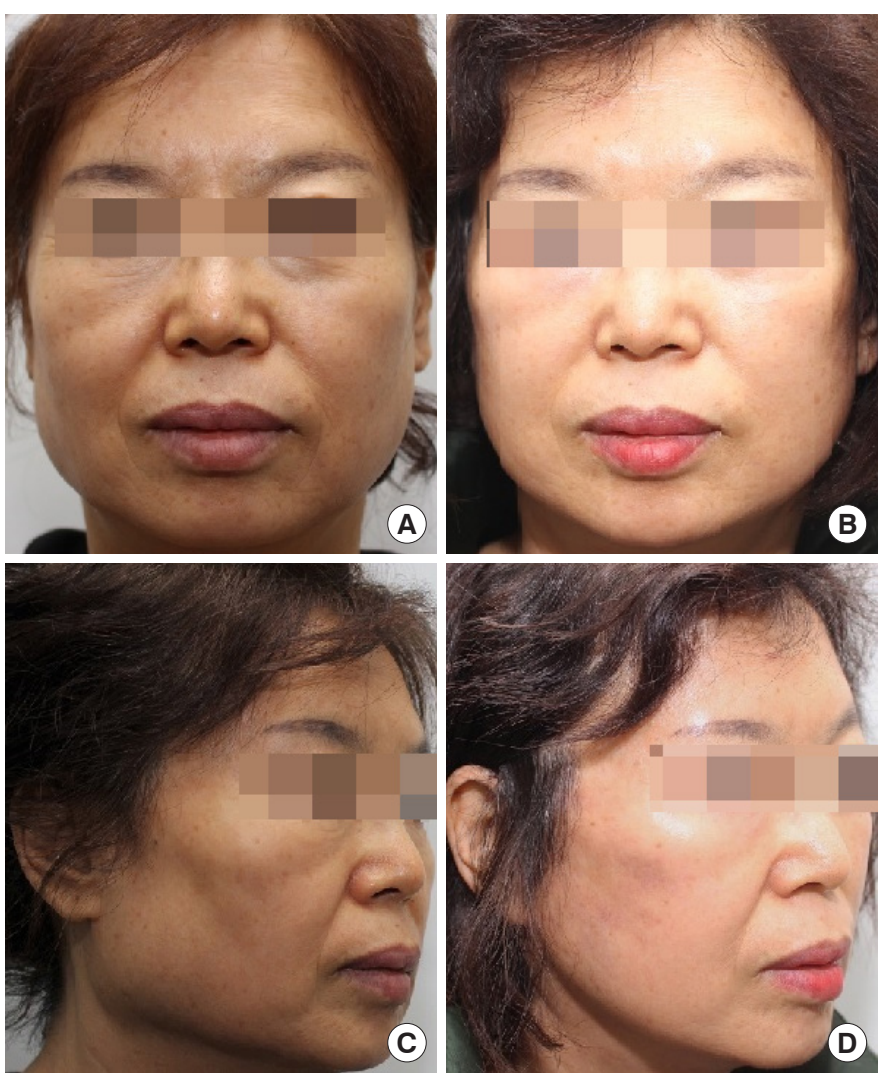

Fig. 5. A 60-year-old woman underwent mandibular angle reduction with facelift surgery. $(A, C)$ Preoperative photograph. (B, D) Postoperative photograph 6 months later, in which the facial rhytides and mandibular contour had significantly improved. Weakening of the nasolabial fold and the change from a rectangular face to an oval face can be observed in the postoperative photograph.

gle area, unlike the malar area, and unfavorable outcomes may be achieved in Asian women with a prominent mandibular angle despite improved facial rhytides. These problems can be solved by simultaneously performing facelift and mandibular angle reduction in Asian women with a prominent mandibular angle.

In general, mandibular angle reduction is performed using the intraoral approach. However, when we perform facelift procedures, the premasseter space is accessible; thus, we could adequately perform mandibular angle reduction. Mendelson presented the concept of the premasseter space. In the sub-SMAS plane, the boundaries of the space are established by the facial retaining ligaments. The premasseter space is located in the lateral lower third of the face between the platysma and the masseter fascia. The lower half of the premasseter space is an avascular cleavage plane, which contains no vital structures. Because this area of the premasseter space does not house any structures and other structures do not pass through this area, safe dissection is possible and the risks of bleeding and facial nerve trauma can be reduced [4].

The main disadvantage of this method for Asian patients is the possibility of hematoma caused by the bleeding that occurs when ostectomy is performed to obtain better results. Therefore, it is necessary to proceed cautiously so that bleeding can be controlled when performing the facelift. The other possible disadvantages include the long operative time, prolonged postoperative swelling of the surgical site, and the steep learning curve. Facial nerve injury may be considered the most significant disadvantage of this surgical technique; however, this has not yet occurred. Using this surgical method, damage to the facial nerve can be avoided by making the line for subperiosteal dissection in the masseter fascia only between the buccal branch and upper mandibular branch of the facial nerve with the naked eye. Furthermore, the incision is located at least $15 \mathrm{~mm}$ above the mandibular rim, comfortably superior to the mandibular branch of the facial nerve.

Compared to mandibular angle reduction requiring an intraoral approach, the recovery time is shorter and the risk of infection is lower. In addition, as the SMAS flap acts as a compression garment, and side effects such as malunion, nonunion, and stepping deformity are rare. To summarize, our surgical method enables a faster recovery by reducing the excess trauma of the surgery in Asian women who want to undergo mandibular angle reduction simultaneously with facelift. Our method also reduces postoperative complications, such as infection and delayed surgical recovery, while producing satisfying results for the patient. In addition, if a surgeon familiarizes himself or herself with the anatomy of the premasseter space and this surgical method through a steep learning curve, mandibular angle reduction can be performed more effectively and easily, with a more intuitive field of view than other surgical methods, by making a direct incision on the mandible safely with no risk of facial nerve injury.

Porcheray et al. [13] used a trans-masseter approach in cervicofacial lifting surgery to perform mandibular angle reduction. In this study, the authors simultaneously performed mandibular angle resection while performing cervicofacial lifting surgery for facial feminization in patients over 50 years of age who underwent maleto-female transition surgery. As in our study, the authors also carefully conducted masseter muscle dissection to approach the mandible while visually checking the buccal branch of facial nerve in the master fascia. As with our surgical methods, Porcheray et al. stated that approaching the mandible by directly dissecting the masseter muscle while avoiding the facial nerve in the premasseter space was not difficult. Their article also confirmed that this method secured a clear view for surgery and was safe from any facial nerve damage. However, further research on the possibility of facial nerve injuries based on a sufficient understanding and a careful anatomical study of the premasseter space seems necessary.

In conclusion, mandibular angle reduction combined with facelift appears effective and safe for Asian women who want to undergo mandibular angle reduction simultaneously with facelift. We achieved high postoperative patient satisfaction and resolved the 
sources of dissatisfaction that may arise when only facelift is performed in Asian patients with a prominent mandibular angle, by performing facelift in combination with facial contouring surgery. This surgical method may help Asian women regain their youthful appearance safely and effectively.

\section{NOTES}

\section{Conflict of interest}

No potential conflict of interest relevant to this article was reported.

\section{Ethical approval}

This study was approved by the Institutional Review Board of Eulji University Hospital (IRB No. EMC 2019-12-004-001) and performed in accordance with the principles of the Declaration of Helsinki.

\section{Patient consent}

The patients provided written informed consent for the publication and the use of their images.

\section{ORCID}

Yoon Joo Lee

https://orcid.org/0000-0003-4915-8282

Il Seok Lee

https://orcid.org/0000-0002-8313-2513

Ho Jik Yang

https://orcid.org/0000-0003-0839-780X

\section{REFERENCES}

1. Collawn SS, Vasconez LO, Gamboa M, et al. Subcutaneous approach for elevation of the malar fat pad through a prehairline incision. Plast Reconstr Surg 1996;97:836-41.

2. Pessa JE. An algorithm of facial aging: verification of Lambros's theory by three-dimensional stereolithography, with reference to the patho- genesis of midfacial aging, scleral show, and the lateral suborbital trough deformity. Plast Reconstr Surg 2000;106:479-88.

3. Owsley JQ, Roberts CL. Some anatomical observations on midface aging and long-term results of surgical treatment. Plast Reconstr Surg 2008;121:258-68.

4. Mendelson BC, Freeman ME, Wu W, et al. Surgical anatomy of the lower face: the premasseter space, the jowl, and the labiomandibular fold. Aesthetic Plast Surg 2008;32:185-95.

5. Bartlett SP, Grossman R, Whitaker LA. Age-related changes of the craniofacial skeleton: an anthropometric and histologic analysis. Plast Reconstr Surg 1992;90:592-600.

6. Shaw RB Jr, Kahn DM. Aging of the midface bony elements: a threedimensional computed tomographic study. Plast Reconstr Surg 2007;119:675-81.

7. Onizuka T, Hosaka Y, Miyata M, et al. Our mini-facelift for Orientals. Aesthetic Plast Surg 1995;19:49-58.

8. Gu Y, McNamara JA Jr, Sigler LM, et al. Comparison of craniofacial characteristics of typical Chinese and Caucasian young adults. Eur J Orthod 2011;33:205-11.

9. Coleman SR. Facial augmentation with structural fat grafting. Clin Plast Surg 2006;33:567-77.

10. Burres S. Midface volume replacement with a transmaxillary implant. Aesthetic Plast Surg 2005;29:1-4.

11. Barton FE Jr, Hunt J. The high-superficial musculoaponeurotic system technique in facial rejuvenation: an update. Plast Reconstr Surg 2003;112:1910-7.

12. Stuzin JM. Restoring facial shape in face lifting: the role of skeletal support in facial analysis and midface soft-tissue repositioning. Plast Reconstr Surg 2007;119:362-76.

13. Porcheray M, Bachelet JT, Brosset S, et al. Mandibular angle resection using cervicofacial lifting surgical approach: technical note. J Stomatol Oral Maxillofac Surg 2020;121:434-8. 